\title{
Reviews
}

\section{Deutsches Fremdwörterbuch, Band 6: Gag - Gynäkologie}

Völlig neubearbeitet im Institut für Deutsche Sprache von Gerhard Strauß, Herbert Schmidt, Dominik Brückner, Isolde Nortmeyer, Oda Vietze. Unter Mitarbeit von Heidrun Kämper. XIV/692 S. - Berlin / New York: de Gruyter, 2008, 2. Auflage. ISBN: 978-3-11-019457-9, Preis: 238,00 EUR

Vier Jahre nach Band 5 (erschienen 2004) ist dieser 6. Band erschienen, in dessen Erarbeitungszeit der langjährige Leiter und konzeptionelle Kopf des Projekts „Deutsches Fremdwörterbuch - Neubearbeitung“, Gerhard Strauß, verstarb. Natürlich wird ein solches ,Generationenprojekt" fortgesetzt, nun unter neuer Leitung (Herbert Schmidt), aber im Wesentlichen mit der erfahrenen Mann- bzw. Frauschaft, deren Mitglieder im Titel des Bands aufgeführt sind. Trotz weitgehender personeller Kontinuität stellt sich in solchen Situationen die Frage nach „Kontinuität“ einerseits und „Veränderung“ andererseits (Schmidt 2009). Regelmäßig wird diese Frage mit einem Sowohl-als-auch beantwortet; man muss also näher hinschauen.

Die im Vorwort von Band 6 und in Schmidt 2009 genannten Veränderungen sind durchweg positiv zu nennen und haben auf den Inhalt der Wortstrecke wie auf deren Qualität keinen belegbaren, schon gar keinen negativen Einfluss. Sie beziehen sich vielmehr auf organisatorische Elemente, die das Deutsche Fremdwörterbuch in seiner Gesamtheit, nicht nur in diesem Band, den Interessen heutiger Nutzerinnen und Nutzer deutlich näher bringen. Hier sind v.a. folgende zu nennen:

(1) Das aktualisierte und nach Kurzformeln sortierte Zweitquellen- und Sekundärliteraturverzeichnis (S. VI bis XIV) vereindeutigt die Herkunft der lexikografischen Informationen.

(2) Die Basis der historischen Primärquellen mit originalen Verwendungskontexten der Lemmata und Sublemmata ist aufgrund der in den letzten Jahren immens gestiegenen, im Internet frei zugänglichen Digitalisierung auch historischen Textmaterials enorm angewachsen. Das auf S. XIV des Bands gelistete Material (überwiegend auf CD-Rom veröffentlicht) ist längst nicht das einzige, auf das die DFWB-LexikografInnen inzwischen zugreifen. Neben eigenem historischen Korpusmaterial steht eine unabgeschlossene Menge potenziell nutzbarer Texte im weltweiten Netz. In Brückner 2009 geht der DFWB-Mitarbeiter akribisch der Frage nach, inwieweit die vielfach beklagte Raubdigitalisierungspraxis des Konzerns Google für die historische Wortforschung genutzt werden kann. Dass damit die Quellenbasis nicht nur viel größer, sondern womöglich zu einer unabschließbaren Menge von Titeln wird, hat erhebliche Auswirkungen auf den o.g. Punkt (1). Je mehr Internet-Quellen genutzt werden, desto schneller tritt der Fall ein, dass ein historisches Belegwörterbuch wie 
das DFWB den Nutzern seine Quellen nicht mehr vollständig benennen kann. Damit werden aber auch Untersuchungen zur Frage der Textsortenstreuung, der (v.a. fachlichen) Varietäten, der mehr oder weniger breiten Repräsentation historischer Sprachstufen des Wörterbuchs unmöglich.

Ganz unabhängig vom konkreten Nutzen für dieses Wörterbuch-Projekt kann man hier also die Entstehung eines gewissermaßen neuen offenen und dynamischen Korpustyps beobachten. Damit verschwindet aber auch die Möglichkeit, die Grundgesamtheit einer im Wörterbuch oder sonstwo zu beschreibenden historischen oder sonstigen Varietät zu bestimmen, deren ,Repräsentation“ oder besser: Exemplifizierung das betreffende Korpus darstellt. Ohne Frage stellt dies eine methodische Schwachstelle dar, insofern es unmöglich wird, die Recherche exakt zu wiederholen; die intersubjektive Plausibilität einer Lexemgeschichte nimmt durch extensive Nutzung der Fülle des Internets zweifellos ab. Dennoch wäre es falsch, die neuen Möglichkeiten zu beklagen und aus methodischen Gründen abzulehnen, sondern es gilt, die methodischen Standards anders als auf herkömmliche Art und Weise, aber doch zu wahren. So spräche einiges dafür, die Vielzahl der in einem Projekt bzw. bei einer Lexemanalyse genutzten Primärquellen aus dem Internet durch Nennung der URLs aufzulisten und wenn möglich durch Kommentierung der jeweiligen Inhalte zu charakterisieren. Etwas ist hier auf jeden Fall besser als nichts.

(3) Bei den Wortgeschichten gehen die Autoren nun ggf. bis ins Mittel- und Althochdeutsche zurück, das ist konsequent, denn Entlehnungsvorgänge ins Deutsche gab es bekanntlich nicht erst seit dem 15. Jahrhundert, sondern von Anfang an.

(4) Bei Personenbezeichnungen werden jetzt auch die movierten Feminina berücksichtigt. In den Belegblöcken (z.B. von Geodät/-in; Gouverneur/Gouvernatrix) wird zwar nicht zwischen maskuliner und femininer Benennung unterschieden, aber Interessierte können aus der Belegchronologie (oder aus dem Artikelkopf) offenbar den Erstbeleg des Femininums (bei Geodätin, genauer: GeodätIn mit Binnen-Majuskel: 1999) erschließen. Bei Gelegenheit wäre man für nähere Informationen über die Prinzipien der Behandlung movierter Personenbezeichnungen dankbar, denn in zahlreichen Fällen (z.B. Apothekerin) hat das Femininum im Verlauf der Geschichte einen denotativen Bruch von ,Ehefrau des die Tätigkeit bzw. den Beruf ausübenden Mannes` zu , selbständige Akteurin ...' erfahren und auch die Pragmatik bzw. der kulturhistorische Kontext ist bei Maskulinum und Femininum nicht selten deutlich unterschieden.

(5) Stark erweitert, außerordentlich informativ und in gut überlegter Komplementarität zum gedruckten Text angelegt ist inzwischen das Angebot der Projektwebsite (http://www.idsmannheim.de/ll/fremdwort/). In Ergänzung zum gedruckten Buch werden hier wertvolle Hilfen geboten, und zwar zu den bisherigen wie auch zu den künftigen Bänden der Neubearbeitung. So kann man die geplante Stichwortstrecke einsehen und sogar Ergänzungswünsche äußern. Insgesamt trägt das traditionsreiche DFWB damit dem inzwischen üblich gewordenen Rezeptionsverhalten der Internet-Generation Rechnung, die punktuelle und rasche Informationsrecherche, immer weniger aber langsame Lektüre auf sich zu nehmen bereit ist.

(6) Veränderte Arbeitsorganisation. Normalerweise erfährt man zu solchen Vorgehensweisen nichts. Umso löblicher ist die hier geübte Transparenz (siehe Vorwort, S. V; Schmidt 2009, 
19). Die organisatorischen Änderungen, die ab diesem 6. Band eingeführt wurden - v.a. eine stärkere Teamorientierung bei gleichzeitiger „,vereinheitlichender“ ,Endredaktion“ (ebd.) - lassen aber kaum erkennen, ob sie eher der Qualität oder eher dem Tempo des ,Outputs' zugutekommen sollen. Konsequenterweise wird nun auf die zuvor übliche namentliche Kennzeichnung der Artikel, d.h. auf eine individuelle Autorschaft verzichtet.

Leider führt eine lange Bearbeitungsdauer, wie sie bei großen wissenschaftlichen lexikografischen Projekten bis zu einem gewissen Grad die notwendige Regel ist, heutzutage zu einem weiteren, eher neuen Problem: Die Recherche- und Medienrezeptionsgewohnheiten der jüngeren Generationen haben sich drastisch gewandelt. Die Kompetenz des lesenden Dekodierens solch besonderer Texte, wie längere, mehr oder weniger stark verdichtete Wörterbuchartikel sie darstellen, hat abgenommen und nimmt weiter ab. Dies gilt auch im vorliegenden Fall, obwohl gerade das DFWB sich von Anfang an durch eine gute Lesbarkeit auszeichnete, nicht zuletzt weil sie zu den expliziten konzeptionellen Merkmalen der Neubearbeitung gehörte:

„Die Artikel mit ihrem vielfältigen Datenangebot sind nicht im knappen Telegrammstil verfasst, sondern um der besseren Lesbarkeit willen in der Regel ausformuliert. Mit Ausnahme der Verweispfeile $(, \rightarrow$ ") , die die Stichwörter in übergreifende (etymologische und semantische) Wortschatzbezüge einordnen, verzichtet das Fremdwörterbuch weitgehend auf Kommentarsymbole und Abkürzungen als spezifischen Mitteln der lexikographischen Beschreibungs- oder Kommentarsprache - im Unterschied zu den formal stark standardisierten Artikeln der allgemeinsprachlichen Gebrauchswörterbücher. ... Diese Kommentare sollen als Lesehilfen und artikelinterne Benutzungshinweise zugleich verstanden werden. Die Artikel erklären sich somit weitgehend aus sich selbst. Mit der sprachlich ausformulierten Einführung und Identifizierung der Artikelpositionen wird erreicht, dass ein einheitlicher lexikographischer Text entsteht, der trotz der fachlich-linguistischen Ausrichtung im Prinzip aller Informationsarten nur einen geringen Grad an fachlicher Textverdichtung aufweist". (http://www.ids-mannheim.de/ll/fremdwort/artikel/Hinweis.pdf)

Die Syntax der Wortartikel ist aber, nicht erst in diesem 6. Band, insofern exzeptionell, als die Obersätze regelmäßig ohne finite Prädikatsteile auskommen; auch das Fehlen eines formalen Subjekts - hier setzen erfahrene Wörterbuchbuchnutzer stillschweigend das Lemmazeichen ein - erschwert das Verständnis der jeweiligen Proposition sehr. Man kann auch sagen, es handelt sich bei den meisten Obersätzen um sehr komplexe Prädikativum-Konstruktionen mit Ellipse des Subjekts und der Kopula. Die in diese eingebetteten Nebensätze jedoch sind in der Tat ,ausformuliert“ und haben eine standardsprachliche Syntax (vgl. exemplarisch Ghetto, S. 268ff.; Glacé, Grad). Sie bieten oft kulturelle Hintergrundinformationen, die von großem Interesse und für Leser attraktiv sind. M.a.W.: Der Zugang zu den ,Hauptinformationen“ ist schwerer als der zu den ,Nebeninformationen'. Es ist nicht unwahrscheinlich, dass ungeübtere Nutzer deshalb vorrangig die Hintergrund- oder Nebeninformationen wahrnehmen, weniger die für deren Einordnung wesentlicheren Hauptinformationen zur Bedeutungs- oder auch Formentwicklung eines Lexems. Das oben zitierte ,ausformuliert" heißt hier also keineswegs ,syntaktisch vollständig', sondern benennt einen textsortenspezifischen Telegramm-Stil, der hier zwar mit weniger Abkürzungen und nichtsprachlichen Zeichen auskommt als in den meisten Gebrauchswörterbüchern, der aber noch lange nicht einfach lesbar ist. Man muss befürchten, dass die heutige Studierendengeneration und erst recht künftige Generationen, für die Nachschlagewerke besonders leicht und rasch zu konsumieren sein müssen und die kaum noch wörterbuchspezifische Nutzungskompetenzen entwickeln (wollen), die in einem solchen Projekt geleistete Arbeit nicht mehr schätzen werden. 
Das DFWB soll und wird seinen Wörterbuchstil kaum ganz umstellen. Aber wenigstens der Versuch, die verblosen Konstruktionen weniger komplex zu gestalten, könnte einen begeisterten Nutzerkreis noch lange am Leben erhalten.

Die lesende Rezeption der Artikel wird auch durch eine mehr oder weniger gut strukturierte Gliederung der Artikelköpfe beeinflusst; diese hängt natürlich in erster Linie von der Polysemie des betreffenden Lemmazeichens ab. Dennoch ist nicht ganz nachvollziehbar, warum z.B. der Artikelkopf zu Grad besser strukturiert scheint als der zu Geste. Dabei spielt möglicherweise auch eine Rolle, nach welchen Kriterien Kompositabildungen und Ableitungen im laufenden Artikeltext verzeichnet und belegt sind. Wenn der Kommentar „vereinzelt" für nicht-usuelle Bildungen steht - wann ist deren Angabe dann sinnvoll und für Leser informativ? Sicherlich dann, wenn sie eine usuelle Bildung oder Verwendung historisch vorbereiten oder auch das allmähliche Auslaufen ihres Gebrauchs veranschaulichen. Rein okkasionelle Bildungen ohne eine nachvollziehbare Begründung machen den Text des Artikelkopfs noch unübersichtlicher und besitzen kaum Informationswert. So scheint die Angabe zu Gestik (S. 261) sinnvoll und plausibel, die zu goliathisch und Goliatherie (S. 399) aber nicht. Ähnlich wie mit den Wortbildungen verhält es sich mit typischen Syntagmen. Es ist von außen nicht zu erkennen, ob die Markierung als ,typisch“ oder ,üblich' bestimmten Mindestkriterien in der Beleglage entspricht, aber es ist wünschenswert, dass es solche Kriterien gibt.

\section{Literatur}

Brückner, Dominik: Die Google Buchsuche als Hilfsmittel für die Lexikographie. In: Sprachreport 3/2009. S. 26-31.

Schmidt, Herbert: Kontinuität und Veränderung. Zum Erscheinen des 6. Bandes der Neubearbeitung des Deutschen Fremdwörterbuchs (DFWB). In: Sprachreport 1/2009, 17-21. 


\title{
Deutsches Fremdwörterbuch, Band 6: Gag - Gynäkologie
}

\author{
Haß, Ulrike
}

Dieser Text wird über DuEPublico, dem Dokumenten- und Publikationsserver der Universität Duisburg-Essen, zur Verfügung gestellt.

Die hier veröffentlichte Version der E-Publikation kann von einer eventuell ebenfalls veröffentlichten Verlagsversion abweichen.

DOI: https://doi.org/10.1515/lexi.2010.025

URN: urn:nbn:de:hbz:464-20190409-155910-6

Link: https://duepublico.uni-duisburg-essen.de:443/servlets/DocumentServlet?id=48555

Rechtliche Vermerke:

Ulrike Haß dankt dem De Gruyter Verlag für die freundliche Genehmigung, diesen Text online veröffentlichen zu dürfen. Zudem dankt Frau Haß den Herausgebern für die Aufnahme des Beitrags in die Zeitschrift.

Quelle: Erschienen in: Lexicographica. International annual for lexicography. Bd. 21 (2005). S. 108-121. 